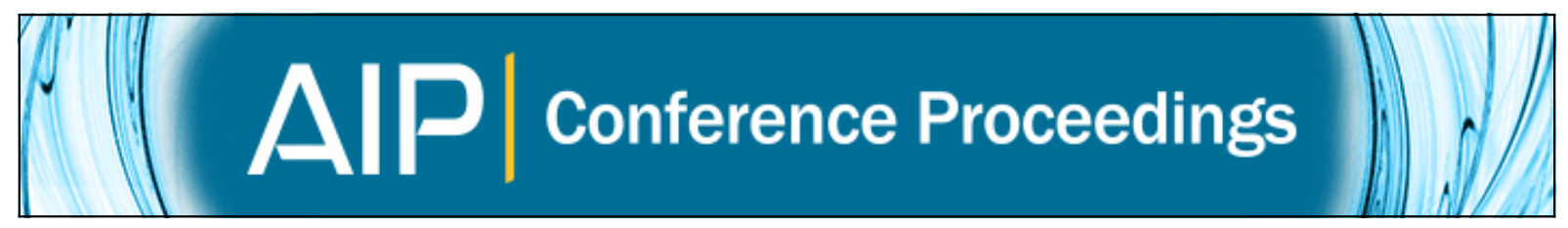

Repairable queue with non-exponential service time and variable breakdown rates

Koh Siew Khew, Pooi Ah Hin, and Tan Yi Fei

Citation: AIP Conference Proceedings 1660, 050026 (2015); doi: 10.1063/1.4915659

View online: http://dx.doi.org/10.1063/1.4915659

View Table of Contents: http://scitation.aip.org/content/aip/proceeding/aipcp/1660?ver=pdfcov

Published by the AIP Publishing

Articles you may be interested in

Origins of the non-exponential reorientation dynamics of nanoconfined water

J. Chem. Phys. 141, 18C523 (2014); 10.1063/1.4896983

Pathway structure determination in complex stochastic networks with non-exponential dwell times

J. Chem. Phys. 140, 184102 (2014); 10.1063/1.4874113

Multi-component non-stationary exponential distributions of the breakdown voltages and time delays in neon ramp breakdown experiments

J. Appl. Phys. 110, 103304 (2011); 10.1063/1.3660687

Structural Heterogeneity and Non-Exponential Relaxation in Supercooled Liquid Silicon

AIP Conf. Proc. 982, 804 (2008); 10.1063/1.2897909

Non-Exponential Decay of Nuclei - A Search for a Fundamental Quantum Effect in Nuclei

AIP Conf. Proc. 680, 305 (2003); 10.1063/1.1619722 


\title{
Repairable Queue with Non-exponential Service Time and Variable Breakdown Rates
}

\author{
Koh Siew Khew ${ }^{\mathrm{a}}$, Pooi Ah Hin ${ }^{\mathrm{b}}$ and Tan Yi Fei \\ ${ }^{a}$ Faculty of Engineering and Science, UTAR, Jalan Genting Kelang, 53300 Setapak, Kuala Lumpur, Malaysia \\ ${ }^{b}$ Sunway University Business School, Sunway University, 47500 Subang Jaya, Selangor, Malaysia \\ ${ }^{c}$ Faculty of Engineering, Multimedia University, 63100 Cyberjaya, Selangor, Malaysia
}

\begin{abstract}
Consider a single server queue in which the service station may breakdown according to a Poisson process with rates $\gamma$ in busy time and $\gamma^{\prime}$ in idle time respectively. After a breakdown, the service station will be repaired immediately and the repair time is assumed to have an exponential distribution with rate $\delta$. Suppose the arrival time has an exponential distribution with rate $\lambda$, and the probability density function $g(t)$ and the cumulative distribution function $G(t)$ of the service time are such that the rate $g(t) /[1-G(t)]$ tends to a constant as $t$ tends to infinity. When the queue is in a stationary state, we derive a set of equations for the probabilities of the queue length and the states of the arrival and service processes. Solving the equations, we obtain approximate results for the stationary probabilities which can be used to obtain the stationary queue length distribution of the system.
\end{abstract}

Keywords: Service time; constant asymptotic rate; stationary queue length distribution.

PACS: 02.50.Ey

\section{INTRODUCTION}

Some queueing systems are unreliable as they may breakdown or fail due to different causes. White and Christie [1] were the first to consider an $\mathrm{M} / \mathrm{M} / 1$ queueing system with the service station subject to exponentially distributed interruptions. Since then, many papers related to server with interruptions were published (see Avi-Itzhak and Naor [2]; Fisher [3]; Vinod and Altiok [4]; Li and Zhu [5]; Yang and Alfa [6]). Most of the authors assumed that the breakdown rate was constant. However, an unreliable server may have different breakdown rates during busy time and idle time. Gray et al. [7] studied a model in which the server takes a vacation when the system is idle, and is subject to breakdown while in operation. Sheng-li et al. [8] considered a model given by an M/M/1 queue in which the service station may break down according to a Poisson process with different rates in idle time and busy time. Sheng-li and Jing-bo [9] extended the study in the model in Sheng-li et al. [8] to multi-state queueing system.

In this paper, we generalize the model in Sheng-li et al. [8] by changing the distribution of the service time to one of which the rate tends to a constant as the time $t$ tends to infinity. Abbreviating "Constant Asymptotic Rate" to "CAR", we may refer to the distribution with a constant asymptotic rate as the CAR distribution. The requirement for the service time distribution to have a constant asymptotic rate is not a great restriction since in practice many distributions such as exponential, Erlang, hyperexponential, gamma, phase-type etc. satisfy this requirement. The resulting queue may now be denoted as an $\mathrm{M} / \mathrm{CAR} / 1$ queue.

To analyze the unreliable M/CAR/1 queue, we first discretize the service time by segmenting the time axis into a sequence of equal intervals of length $\Delta t$. We next derive a set of equations for the stationary probabilities of the queue length and the states of the arrival, service and repair processes. By solving the equations, we obtain approximately the stationary probabilities.

The remainder of the paper is organized as follows. The next section of the paper gives a brief description of the model considered in Sheng-li et al. [8]. Then, in the subsequent section, we derive a set of equations for the stationary probabilities. The method used to solve for the set of equations is presented in the succeeding section. Then, some numerical examples are presented. Concluding remarks of this study are added in the final section. 


\section{MODEL DESCRIPTION}

Sheng-li et al. [8] studied the M/M/1 queue in which the service station may break down according to a Poisson process with rates $\gamma$ in busy time and $\gamma$ ' in idle time respectively. After a breakdown, the service station will be repaired immediately and the repair time is assumed to have an exponential distribution with rate $\delta$. In this paper, we generalize the $\mathrm{M} / \mathrm{M} / 1$ queue to $\mathrm{M} / \mathrm{CAR} / 1$ queue in which the arrival time has an exponential distribution with rate $\lambda$, and the distribution of the service time has a constant asymptotic rate. With the change in the service time distribution, the model may be applied to the system where the service time distribution is non-exponential.

\section{DERIVATION OF EQUATIONS FOR THE STATIONARY PROBABILITIES}

In this section, a set of equations for the stationary probabilities of the queue length and the states of the arrival and service processes in the discretized $\mathrm{M} / \mathrm{CAR} / 1$ queue is derived. First let $g(t)$ be the probability density function (pdf) of the service time and $\tau_{k}$ the interval $((k-1) \Delta t, k \Delta t]$ for $k=1,2,3, \ldots$. Furthermore let

where $I$ is large enough such that

$$
\mu_{k}=\frac{g(k \Delta t)}{\int_{k \Delta t}^{\infty} g(u) d u}, \quad 1 \leq k \leq I,
$$

$$
\mu_{I} \cong \lim _{k \rightarrow \infty} \mu_{k}
$$

Suppose a service starts at time $t=0$. Then the probability that the service will be completed in the interval $\tau_{1}$ is approximately $\mu_{1} \Delta t$, and given that the service is not completed in $\tau_{1}, \tau_{2}, \ldots, \tau_{k-1}$, the probability that the service will be completed in $\tau_{k}$ is approximately $\mu_{k} \Delta t, k=2,3,4, \ldots$ where $\mu_{k}=\mu_{I}$ for $k \geq I$.

Let the interval before $\tau_{1}$ as $\tau_{0}$. Given that a service starts at a time in $\tau_{0}$, we may define the state number $\xi_{k}$ of the service process at the end of $\tau_{k}$ as

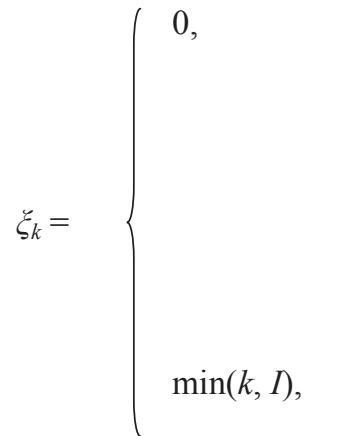

if

- $k=0$; or

- a repair is completed in $\tau_{k}, k \geq 2$; or

- the server is active at the end of $\tau_{k-1}$ and the service ends in $\tau_{k}, k \geq 1$; or

- the server is idle at the end of $\tau_{k-1}$, and in $\tau_{k}$, the server remains idle or a customer arrives or a breakdown occurs, $k \geq 2$.

- the server is active and is at state $\min (k-1, I)$ at the end of $\tau_{k-1}$, and a breakdown occurs in $\tau_{k}, k \geq 1$; or

- the server is being repair at the end of $\tau_{k-1}$ and, no repair completions occur in $\tau_{k}, k \geq 2$.

if the server is active and is in the state $\min (k-1, I)$ at the end of $\tau_{k-1}$, and no breakdown or no service completions occur in $\tau_{k}, k \geq 1$.

Next, given that a customer arrives at a time in $\tau_{0}$, we may define the state number $\psi_{k}$ of the arrival process at the end of $\tau_{k}$ as

$\psi_{k}= \begin{cases}0, & \text { if the next customer does not arrive in } \tau_{k}, k \geq 1 . \\ 1, & \text { if } k=0 \text { or the next customer arrives in } \tau_{k}, k \geq 1 .\end{cases}$

The state number $\varphi_{k}$ of the repair process at the end of $\tau_{k}$ is defined as
$\varphi_{k}=\left\{\begin{array}{c}0, \\ 1,\end{array}\right.$
if
- $k=0$; or
- the server is not broken down at the end of $\tau_{k-1}$ and, no breakdowns occur in $\tau_{k}$; or
- the server is still being repaired at the end of $\tau_{k-1}$ and a repair completion occurs in $\tau_{k}$, $k \geq 2$.
1, if the server is still being repaired at the end of $\tau_{k-1}$ and, no repair completions occur in $\tau_{k}$, $k \geq 2$. 
Let $n_{k}$ be the queue length at the end of $\tau_{k}$ and $\boldsymbol{h}_{k}=\left(n_{k}, \xi_{k}, \varphi_{k}, \psi_{k}\right)$. We may refer to $\boldsymbol{h}_{k}$ as the vector of characteristics of the queue at the end of $\tau_{k}$.

Let $P_{n i j}^{(k)}$ be the probability that at the end of $\tau_{k}$, the number of customers in the system is $n$ (including the customer that is being served), the service process is in state $i$, the repair process is in state $r$ and the arrival process is in state $j$. Assume that

$$
P_{n i j j}=\lim _{k \rightarrow \infty} P_{n i j}^{(k)}
$$

exists. To find the $P_{n i r j}$, we first make the following observations.

Suppose at the end of $\tau_{k-1}$, the queue length $n$ is not empty (i.e. $n_{k-1}=n \geq 1$ ), the server is in state $i-1$, and the arrival process is in state $j$. In this case the server is still active and we define the repair state number to be zero. This means the vector of characteristics at the end of $\tau_{k-1}$ is given by $\boldsymbol{h}_{k-1}=(n, i-1,0, j)$. With this value of $\boldsymbol{h}_{k-1}$, only one of the following events can occur in $\tau_{k}$ :

(a) A customer enters the system with the arrival rate $\lambda$, and at the end of $\tau_{k}$, the vector of characteristics becomes $\boldsymbol{h}_{k}=\left(n+1, i^{*}, 0,1\right)$;

(b) A customer leaves the system with the departure rate $\mu_{i^{*}}$, and $\boldsymbol{h}_{k}=(n-1,0,0,0)$;

(c) The server breaks down, yielding $\boldsymbol{h}_{k}=(n, 0,1,0)$;

(d) No customers enter or leave the system, and no breakdowns occur, yielding $\boldsymbol{h}_{k}=\left(n, i^{*}, 0,0\right)$ where $i^{*}=\min (i, I)$.

However if at the end of $\tau_{k-1}$, the system is empty (i.e. $n_{k-1}=0$ ), the server is not broken down and no customer arrives in $\tau_{k-1}$ with $\boldsymbol{h}_{k-1}=(0,0,0,0)$, then one of the following events can occur in $\tau_{k}$ :

(e) A customer enters the system with arrival rate $\lambda$, and $\boldsymbol{h}_{k}=(1,0,0,1)$;

(f) A breakdown occurs with rate $\gamma^{\prime}$, yielding $\boldsymbol{h}_{k}=(0,0,1,0)$;

(g) No customers enter the system and no breakdowns occur, yielding $\boldsymbol{h}_{k}=(0,0,0,0)$.

Suppose at the end of $\tau_{k-1}$, the queue length is $n_{k-1}=n \geq 0$, the repair process is in state $\varphi_{k-1}=r=1$, and the arrival process is in state $j$. In this case the server is being repaired and we define the service state number to be zero, yielding $\boldsymbol{h}_{k-1}=(n, 0,1, j)$. Then one of the following events can occur in $\tau_{k}$ :

(h) A customer enters the system with arrival rate $\lambda$, and $\boldsymbol{h}_{k}=(n+1,0,1,1)$;

(i) A completion of repair occurs with the repair rate $\delta$, and yielding $\boldsymbol{h}_{k}=(n, 0,0,0)$;

(j) No customers enter the system and no completion of repair occurs, yielding $\boldsymbol{h}_{k}=(n, 0,1,0)$.

By setting $n_{k-1}=0, \xi_{k-1}=0, \varphi_{k-1}=0$ and $\psi_{k-1}=0$ and letting Event (e) occur in $\tau_{k}$, we get

$P_{1001}^{(k)} \cong P_{0000}^{(k-1)}(1-\gamma \Delta t)(\lambda \Delta t)$.

When $k \rightarrow \infty$, we get from (4)

$P_{1001} \cong P_{0000}(1-\gamma \Delta t)(\lambda \Delta t)$.

In general, for a given value of $\boldsymbol{h}_{k}$, we can likewise find the combination of $\boldsymbol{h}_{k-1}$ and the event in $\tau_{k}$ which leads to $\boldsymbol{h}_{k}$, and obtain an equation similar to (5). Thus we can obtain the following equations

$$
\begin{aligned}
& P_{0000} \cong P_{0000}\left(1-\gamma^{\prime} \Delta t\right)(1-\lambda \Delta t)+P_{0010}(\delta \Delta t)+\sum_{m=0}^{I-1} P_{1 m 00}\left(1-\gamma^{\prime} \Delta t\right)\left(\mu_{m+1} \Delta t\right)+P_{1100}\left(1-\gamma^{\prime} \Delta t\right)\left(\mu_{I} \Delta t\right), \\
& \quad+P_{1001}\left(1-\gamma^{\prime} \Delta t\right)\left(\mu_{1} \Delta t\right) \\
& P_{0010} \cong P_{0000}\left(\gamma^{\prime} \Delta t\right)+P_{0010}(1-\delta \Delta t)(1-\lambda \Delta t) .
\end{aligned}
$$

When $n=1$,

$P_{1001} \cong P_{0000}(1-\gamma \Delta t)(\lambda \Delta t)$,

$P_{1100} \cong P_{1000}(1-\gamma \Delta t)\left(1-\mu_{1} \Delta t\right)(1-\lambda \Delta t)+P_{1001}(1-\gamma \Delta t)\left(1-\mu_{1} \Delta t\right)(1-\lambda \Delta t)$,

$P_{1 i 00} \cong P_{1(i-1) 00}(1-\gamma \Delta t)\left(1-\mu_{i} \Delta t\right)(1-\lambda \Delta t)$

for $2 \leq i<I$,

$P_{1 I 00} \cong P_{1(I-1) 00}(1-\gamma \Delta t)\left(1-\mu_{I} \Delta t\right)(1-\lambda \Delta t)+P_{1 I 00}(1-\gamma \Delta t)\left(1-\mu_{I} \Delta t\right)(1-\lambda \Delta t)$,

$P_{1010} \cong \sum_{m=0}^{I} P_{1 m 00}(\gamma \Delta t)+P_{1001}(\gamma \Delta t)+P_{1010}(1-\delta \Delta t)(1-\lambda \Delta t)+P_{1011}(1-\delta \Delta t)(1-\lambda \Delta t)$, 


$$
P_{1011} \cong P_{0010}(1-\delta \Delta t)(\lambda \Delta t)
$$

For $n \geq 1$,

$$
\begin{aligned}
& P_{n 000} \cong P_{n 010}(\delta \Delta t)+P_{n 011}(\delta \Delta t)+\sum_{m=0}^{I-1} P_{(n+1) m 00}(1-\gamma \Delta t)\left(\mu_{m+1} \Delta t\right)+P_{(n+1) I 00}(1-\gamma \Delta t)\left(\mu_{I} \Delta t\right) \\
& \quad+\sum_{m=1}^{I-1} P_{(n+1) m 01}(1-\gamma \Delta t)\left(\mu_{m+1} \Delta t\right)+P_{(n+1) I 01}(1-\gamma \Delta t)\left(\mu_{I} \Delta t\right)
\end{aligned}
$$

When $n=2$,

$$
\begin{array}{ll}
P_{2101} \cong P_{1000}(1-\gamma \Delta t)\left(1-\mu_{1} \Delta t\right)(\lambda \Delta t)+P_{1001}(1-\gamma \Delta t)\left(1-\mu_{1} \Delta t\right)(\lambda \Delta t), & \\
P_{2 i 01} \cong P_{1(i-1) 00}(1-\gamma \Delta t)\left(1-\mu_{i} \Delta t\right)(\lambda \Delta t) & \text { for } 2 \leq i<I, \\
P_{2 I 01} \cong P_{1(I-1) 00}(1-\gamma \Delta t)\left(1-\mu_{I} \Delta t\right)(\lambda \Delta t)+P_{1100}(1-\gamma \Delta t)\left(1-\mu_{I} \Delta t\right)(\lambda \Delta t) . &
\end{array}
$$

For $n \geq 2$,

$$
\begin{aligned}
& P_{n 100} \cong P_{n 000}(1-\gamma \Delta t)\left(1-\mu_{1} \Delta t\right)(1-\lambda \Delta t), \\
& P_{n i 00} \cong P_{n(i-1) 00}(1-\gamma \Delta t)\left(1-\mu_{i} \Delta t\right)(1-\lambda \Delta t)+P_{n(i-1) 01}(1-\gamma \Delta t)\left(1-\mu_{i} \Delta t\right)(1-\lambda \Delta t) \\
& P_{n I 00} \cong P_{n(I-1) 00}(1-\gamma \Delta t)\left(1-\mu_{I} \Delta t\right)(1-\lambda \Delta t)+P_{n(I-1) 01}(1-\gamma \Delta t)\left(1-\mu_{I} \Delta t\right)(1-\lambda \Delta t) \\
& \quad+P_{n I 00}(1-\gamma \Delta t)\left(1-\mu_{I} \Delta t\right)(1-\lambda \Delta t)+P_{n I 01}(1-\gamma \Delta t)\left(1-\mu_{I} \Delta t\right)(1-\lambda \Delta t) \\
& P_{n 010} \cong \sum_{m=0}^{I} P_{n m 00}(\gamma \Delta t)+\sum_{m=1}^{I} P_{n m 01}(\gamma \Delta t)+P_{n 010}(1-\delta \Delta t)(1-\lambda \Delta t)+P_{n 011}(1-\delta \Delta t)(1-\lambda \Delta t), \\
& P_{n 011} \cong P_{(n-1) 010}(1-\delta \Delta t)(\lambda \Delta t)+P_{(n-1) 011}(1-\delta \Delta t)(\lambda \Delta t) .
\end{aligned}
$$

For $n \geq 3$,

$$
\begin{aligned}
& P_{n 101} \cong P_{(n-1) 000}(1-\gamma \Delta t)\left(1-\mu_{1} \Delta t\right)(\lambda \Delta t), \\
& P_{n i 01} \cong P_{(n-1)(i-1) 00}(1-\gamma \Delta t)\left(1-\mu_{i} \Delta t\right)(\lambda \Delta t)+P_{(n-1)(i-1) 01}(1-\gamma \Delta t)\left(1-\mu_{i} \Delta t\right)(\lambda \Delta t)
\end{aligned}
$$

and

$$
\begin{aligned}
& P_{n I 01} \cong P_{(n-1)(I-1) 00}(1-\gamma \Delta t)\left(1-\mu_{I} \Delta t\right)(\lambda \Delta t)+P_{(n-1)(I-1) 01}(1-\gamma \Delta t)\left(1-\mu_{I} \Delta t\right)(\lambda \Delta t) \\
& \quad+P_{(n-1) I 00}(1-\gamma \Delta t)\left(1-\mu_{I} \Delta t\right)(\lambda \Delta t)+P_{(n-1) I 01}(1-\gamma \Delta t)\left(1-\mu_{I} \Delta t\right)(\lambda \Delta t)
\end{aligned}
$$

\section{STATIONARY QUEUE LENGTH DISTRIBUTION}

In this section, the method in Koh [10] shall be used to solve equations (6) to (25) in order to obtain the stationary queue length distribution. First let $b_{i j}, c_{j}, d_{i j}, e_{j}, f_{i j}, h_{r}$ and $v_{i j}$ be constants and introduce the following notations:

1) $P_{n^{* * *}}=\left\{P_{n i r}: 0 \leq i \leq I, r=0,1\right.$ and $\left.j=0,1\right\}$;

2) $\quad P_{n^{*} 0^{*}}=\left\{P_{n i 0 j}: 0 \leq i \leq I, j=0,1\right\}$;

3) $\left(P_{m^{* * *}}, P_{(m+1)^{* * *},} P_{\left.(m+2)^{*} 0^{*}\right)}\right.$ denotes the set of equations of the form

$$
\sum_{i=0}^{I} \sum_{j=0}^{1} b_{i j} P_{m i 0 j}+\sum_{j=0}^{1} c_{j} P_{m 01 j}+\sum_{i=0}^{I} \sum_{j=0}^{1} d_{i j} P_{(m+1) i 0 j}+\sum_{j=0}^{1} e_{j} P_{(m+1) 01 j}+\sum_{i=0}^{I} \sum_{j=0}^{1} f_{i j} P_{(m+2) i 0 j} \cong 0 ;
$$


4)

$$
\begin{aligned}
& \left(P_{m i r j} \mid P_{00^{*} 0}, P_{(m+1)^{*} * 0^{*}}\right) \text { denotes the equation of the form } \\
& P_{m i r j} \cong \sum_{r=0}^{1} h_{r} P_{00 r 0}+\sum_{i=0}^{I} \sum_{j=0}^{1} v_{i j} P_{(m+1) i 0 j} .
\end{aligned}
$$

With the above notations, (8) to (14) in the case when $n=1$ can be represented as

$$
\left(P_{0 * *}, P_{1 * * *}, P_{2^{*} 0 *}\right)
$$

and (14) to (22) in the case when $n=2$ may be represented as

$$
\left(P_{1 * * *}, P_{2^{* * *},}, P_{3^{*} 0 *}\right)
$$

When $n \geq 3$, (14) together with (18) to (25) can be represented as

$$
\left(P_{(n-1)^{* * *}}, P_{n^{* * *}}, P_{\left.(n+1)^{*} 0^{*}\right)}\right.
$$

It can be shown that from the set of equations given by (26), we can get

$$
\left(P_{1 i r j} \mid P_{00^{*} 0}, P_{2^{*} 0^{*}}\right) \quad \text { for } 0 \leq i \leq I, r=0,1 \text { and } j=0,1 \text {. }
$$

By substituting the expression of the $P_{1 i r j}$ given by (29) into (27), and solving for the $P_{2 i r j}$, we get

$$
\left(P_{2 i r j} \mid P_{00^{*} 0}, P_{3^{*} 0^{*}}\right) \quad \text { for } 0 \leq i \leq I, r=0,1 \text { and } j=0,1 \text {. }
$$

By substituting the expression of the $P_{2 i r j}$ given by (30) into (28) when $n=3$ and solving for the $P_{3 i r j}$, we get

$$
\left(P_{3 i r j} \mid P_{00^{*} 0}, P_{4^{*} 0^{*}}\right) \quad \text { for } 0 \leq i \leq I, r=0,1 \text { and } j=0,1 .
$$

Next for $n \geq 4$, repeat the process of substituting the expression of the $P_{(n-1) i r j}$ given by

$$
\left(P_{(n-1) i r j} \mid P_{00^{*} 0}, P_{n^{*} 0^{*}}\right) \quad \text { for } 0 \leq i \leq I, r=0,1 \text { and } j=0,1
$$

into (28) and solving for the $P_{\text {nirj }}$ to get

$$
\left(P_{n i r j} \mid P_{00^{*} 0}, P_{\left.(n+1)^{*} 0^{*}\right)} \quad \text { for } 0 \leq i \leq I, r=0,1 \text { and } j=0,1 .\right.
$$

When $n=N$ is large enough, we may set all the $P_{(n+1)^{*} 0^{*}}$ in (33) to be zero and obtain

$$
\left(P_{n i r j} \mid P_{00 * 0}\right) \quad \text { for } 0 \leq i \leq I, r=0,1 \text { and } j=0,1 \text {. }
$$

For $n=N-1, N-2, \ldots, 1$, we may perform the substitution of $\left(P_{(n+1) i r j} \mid P_{00 * 0}\right)$ into (33) and obtain

$$
\left(P_{n i r j} \mid P_{00 * 0}\right) \quad \text { for } 0 \leq i \leq I, r=0,1 \text { and } j=0,1 \text {. }
$$

When $n=1$, (35) yields $\left(P_{1 i r j} \mid P_{00^{*} 0}\right)$. By using the results given by $\left(P_{1 i r j} \mid P_{00^{*} 0}\right)$ and the Equations (6) and (7), we get the following system of two equations:

$$
\left(P_{00 * 0} \mid P_{00 * 0}\right) \quad \text { for } r=0,1 .
$$

An inspection of (36) reveals that the two equations are linearly dependent. Hence, we need to include another linearly independent equation so that the resulting system of two equations has a unique solution. Equating the sum of the left sides of the equations given by (35) to the sum of the right sides of (35), we get an equation of the form,

$$
\sum_{n=1}^{N} \sum_{i} \sum_{r} \sum_{j} P_{n i r j}=\sum_{r} k_{r} P_{00 r 0}
$$

where $r=0,1$ and the $k_{r}$ are constants.

As $\sum_{n=1}^{N} \sum_{i} \sum_{r} \sum_{j} P_{n i r j} \cong 1$, we get from (37) an equation involving only $P_{00 r 0}, r=0,1$. This equation derived from (37), and one equation chosen from (36), constitute a system of two equations which can be solved to yield numerical answers for $P_{00 r 0}, r=0,1$. Then using (35), we can get numerical answers for $P_{n i r j}$ where $n \geq 1$, $0 \leq i \leq I, r=0,1$ and $j=0,1$. The stationary probability that the queue length is $n$ is then given by the sum of the 


\section{NUMERICAL EXAMPLE}

TABLE 1 shows the results of the steady-state mean system size when the service time is exponentially distributed and $\lambda=1$. The numerical results are compared with those given in Sheng-li et al. [8]. Simulation has been carried out to verify the results obtained.

TABLE (1). Comparison of results obtained by the proposed numerical method and those given in Sheng-li et al. [8] and simulation procedure $\left[\Delta t=10^{-9}\right.$ for mean system size, $\left.\mu_{i}=\mu, I=3\right]$.

\begin{tabular}{|c|c|c|c|}
\hline \multirow{2}{*}{ Service rate, $\mu$} & \multicolumn{3}{|c|}{ Mean system size $\left[\gamma=0.1, \gamma^{\prime}=0, \delta=1.2\right]$} \\
\hline & Numerical method & Sheng-li et al. [8] & Simulation \\
\hline 1.1 & 69.1667 & 69.1789 & 63.5311 \\
\hline 1.3 & 5.3205 & 5.3205 & 5.2272 \\
\hline 1.5 & 2.7667 & 2.7667 & 2.7451 \\
\hline 1.7 & 1.8693 & 1.8694 & 1.8688 \\
\hline 1.9 & 1.4116 & 1.4116 & 1.4056 \\
\hline \multirow{2}{*}{ Service rate, $\mu$} & \multicolumn{3}{|c|}{ Mean system size $\left[\gamma=0.2, \gamma^{\prime}=0.1, \delta=2.2\right]$} \\
\hline & Numerical method & Sheng-li et al. [8] & Simulation \\
\hline 1.1 & 124.6259 & 124.5650 & 125.8377 \\
\hline 1.3 & 5.4348 & 5.4348 & 5.4683 \\
\hline 1.5 & 2.7874 & 2.7874 & 2.7564 \\
\hline 1.7 & 1.8787 & 1.8787 & 1.8775 \\
\hline 1.9 & 1.4191 & 1.4192 & 1.4162 \\
\hline \multirow{2}{*}{ Service rate, $\mu$} & \multicolumn{3}{|c|}{ Mean system size $\left[\gamma=0.1, \gamma^{\prime}=0.2, \delta=1.4\right]$} \\
\hline & Numerical method & Sheng-li et al. [8] & Simulation \\
\hline 1.1 & 39.3772 & 39.3750 & 38.1960 \\
\hline 1.3 & 5.0000 & 5.0000 & 4.9727 \\
\hline 1.5 & 2.7083 & 2.7083 & 2.7147 \\
\hline 1.7 & 1.8750 & 1.8750 & 1.8649 \\
\hline 1.9 & 1.4440 & 1.4440 & 1.4420 \\
\hline
\end{tabular}

TABLE 1 shows that results based on the proposed numerical method are very close to those given in Sheng-li et al. [8] and the simulation procedure. Next consider the examples in which the service time has a gamma distribution. The stationary queue length distribution and mean system size are found. The results obtained are shown in TABLE 2 and 3. 
TABLE (2). Comparison of results computed using the proposed numerical method, and simulation procedure $\left[(\kappa, \theta)=(1.5,2.5), \lambda=0.1, \delta=0.3, \gamma=0.05, \gamma^{\prime}=0.03\right][\Delta t=0.04$ for service time distribution, $I=500, N=250]$.

\begin{tabular}{ccc}
\hline \multirow{2}{*}{ Queue Size, $\boldsymbol{n}$} & \multicolumn{2}{c}{ P(Queue Size $=\boldsymbol{n})$} \\
\cline { 2 - 3 } & Numerical method & Simulation \\
\hline 0 & 0.541201 & 0.541048 \\
2 & 0.256167 & 0.256268 \\
3 & 0.112564 & 0.112567 \\
4 & 0.049746 & 0.049747 \\
5 & 0.022198 & 0.022195 \\
6 & 0.009962 & 0.009972 \\
7 & 0.004483 & 0.004493 \\
8 & 0.002020 & 0.002034 \\
9 & $9.11 \mathrm{E}-04$ & $9.15 \mathrm{E}-04$ \\
10 & $4.11 \mathrm{E}-04$ & $4.21 \mathrm{E}-04$ \\
$\ldots$ & $1.85 \mathrm{E}-04$ & $1.85 \mathrm{E}-04$ \\
50 & $\ldots$ & $\ldots$ \\
\hline
\end{tabular}

TABLE (3). Comparison of results computed using the proposed numerical method, and simulation procedure $\left[(\kappa, \theta)=(2,1.5), \lambda=0.2, \delta=0.3, \gamma=0.05, \gamma^{\prime}=0.03\right][\Delta t=0.03$ for service time distribution, $I=500, N=500]$

\begin{tabular}{ccc}
\hline \multirow{2}{*}{ Queue Size, $\boldsymbol{n}$} & \multicolumn{2}{c}{$\mathbf{P}(\mathbf{Q u e u e}$ Size $=\boldsymbol{n})$} \\
\cline { 2 - 3 } & Numerical method & Simulation \\
\hline 0 & 0.271988 & 0.271920 \\
2 & 0.217738 & 0.218206 \\
3 & 0.151907 & 0.152203 \\
4 & 0.105408 & 0.105413 \\
5 & 0.073848 & 0.073745 \\
6 & 0.052096 & 0.052055 \\
7 & 0.036882 & 0.036710 \\
8 & 0.026154 & 0.025998 \\
9 & 0.018559 & 0.018461 \\
10 & 0.013174 & 0.013120 \\
$\ldots$ & 0.009352 & 0.009342 \\
50 & $\ldots$ & $\ldots$ \\
\hline
\end{tabular}

Tables 2 and 3 show that the results obtained using the proposed numerical method are close to the simulation results.

\section{CONCLUSION}

In this paper, a more general version of the $\mathrm{M} / \mathrm{M} / 1$ queue given in Sheng-li et al. [8] is studied. The service time in the model is changed to one which has a fairly general distribution called the CAR distribution. The numerical method proposed in Koh [10] has been successfully used for finding the stationary queue length distribution. Theoretically, the numerical method may also be used to study other even more general queueing system in which both the service time and arrival time follow the CAR distribution. 


\section{REFERENCES}

[1] H. C. White and L. S. Christie, Operations Research 6, 79-95 (1958).

[2] B. Avi-Itzhak and P. Naor, Operations Research 11(3), 303-322 (1963).

[3] M. J. Fischer, Management Science 24(3), 338-344 (1977).

[4] B. Vinod and T. Altiok, The Journal of the Operational Research Society 37(3), 309-316 (1986).

[5] H. Li and Y. Zhu, Journal of Applied Probability 31(4), 1083-1097 (1994).

[6] X. L. Yang and A. S. Alfa, Computers \& Industrial Engineering 56(1), 33-43 (2009).

[7] W. J. Gray, P. P. Wang and M. Scott, Applied Mathematical Modelling 24(5-6), 391-400 (2000).

[8] L. V. Sheng-li, L. Jing-bo and Y. De-quan, "The $M / M / 1$ repairable queueing system with variable breakdown rates" in Control and Decision Conference, 2009. Conference Proceedings, IEEE, Guilin, China, 2009, pp. 2635-2637.

[9] L. V. Sheng-li and L. Jing-bo, Discrete Dynamics in Nature and Society 2013, 1-10 (2013).

[10] S. K. Koh, "Maintenance of Deteriorating Non-Exponential Single Server Queue", Ph.D. Thesis, Universiti Malaya, 2013. 